\title{
Criminologie
}

\section{Le droit pénal de l'environnement : une garantie d'impunité ?}

\section{Maryse Grandbois}

Volume 21, numéro 1, 1988

Entre les mailles de la loi : pollution, accident de travail, faute professionnelle

URI : https://id.erudit.org/iderudit/017258ar

DOI : https://doi.org/10.7202/017258ar

Aller au sommaire du numéro

\section{Éditeur(s)}

Les Presses de l'Université de Montréal

ISSN

0316-0041 (imprimé)

1492-1367 (numérique)

Découvrir la revue

Citer cet article

Grandbois, M. (1988). Le droit pénal de l'environnement : une garantie d'impunité ? Criminologie, 21(1), 57-81. https://doi.org/10.7202/017258ar

\section{Résumé de l'article}

The limits of pollution authorized by law are constantly being exceeded in Quebec and throughout Canada, as evidenced by acid rain, the disposal of dangerous refuse in nature and the uncontrolled use of pesticides. The criminal law on the environment exists, to be sure, but its application is highly relative. In principle, certain infractions under the Criminal Code can be used against polluters but the State rarely makes use of them. As to the statutory criminal law -the main subject of this article - the author notes that it suffers from a lack of clarity and precision. Complex and unorganized, it is characterized by a multitude of infractions and a disparity of sanctions. However, the fact is that the public administration very seldom resorts to the provisions of this law to have industrial activities conform to the laws and regulations. When it does do so, very often the courts hesitate to impose sanctions of a truly deterrent nature. In spite of all this, the fact is that the main obstacle to the criminal law on the environment is the lack of firm determination on the part of the legislators and public administrations to use severe measures against polluters. As long as this political will is lacking, notes the author, certain major industrials will continue to contaminate the environment with impunity. 


\section{LE DROIT PÉNAL DE L'ENVIRONNEMENT: UNE GARANTIE D'IMPUNITÉ? \\ Maryse Grandbois*}

The limits of pollution authorized by law are constantly being exceeded in Quebec and throughout Canada, as evidenced by acid rain, the disposal of dangerous refuse in nature and the uncontrolled use of pesticides. The criminal law on the environment exists, to be sure, but its application is highly relative. In principle, certain infractions under the Criminal Code can be used against polluters but the State rarely makes use of them. As to the statutary criminal law the main subject of this article - the author notes that it suffers from a lack of clarity and precision. Complex and unorganized, it is characterized by a multitude of infractions and a disparity of sanctions. However, the fact is that the public administration very seldom resorts to the provisions of this law to have industrial activities conform to the laws and regulations. When it does do so, very often the courts hesitate to impose sanctions of a truly deterrent nature. In spite of all this, the fact is that the main obstacle to the criminal law on the environment is the lack of firm determination on the part of the legislators and public administrations to use severe measures against polluters. As long as this political will is lacking, notes the author, certain major industrials will continue to contaminate the environment with impunity.

\section{INTRODUCTION : LES CRIMINALITÉS DE L'ENVIRONNEMENT}

L'état général de l'environnement, au Canada, ne s'est pas réellement amélioré, ni même maintenu à ses niveaux antérieurs depuis que les États fédéral et provincial se sont dotés d'un droit de l'environnement, au début des années 19701.

Dans certains secteurs d'activité industrielle, par exemple, les atteintes à l'environnement se font même de plus en plus nombreuses et causent chaque année des dommages importants, autant aux biens collectifs qu'à la propriété privée. Il en va ainsi pour les précipitations

* Avocate et professeure au Département des sciences juridiques, Université du Québec à Montréal, C.P. 8888, succ. «A», Montréal, Qc, H3C 3P8.

1. Ce sont les conclusions d'une étude générale, réalisée par Environnement Canada en 1986. L'État de l'environnement au Canada, Environnement Canada, Ottawa, 1986. 
acides $^{2}$ dont on parle tant. Alimentées principalement par les émissions de soufre et d'azote des hautes cheminées d'usine, ces pluies acides dégradent lentement les ressources naturelles et les matériaux, en plus de constituer une menace pour la santé.

Le Sud du Québec reçoit à lui seul, plus de 20 kilogrammes par hectare, par année de précipitations acides. Actuellement, on sait que cela signifie une diminution des populations de saumons de l'Atlantique dans plusieurs cours d'eau, l'affaiblissement de la densité et de la diversité des espèces, ainsi que l'acidification des eaux souterraines peu profondes ${ }^{3}$. De sorte que la croissance des forêts ralentit, le feuillage des végétaux s'endommage et les récoltes diminuent ${ }^{4}$.

Rappelons au passage que la pêche sportive, le tourisme et l'industrie forestière, principaux secteurs économiques menacés à court terme, représentent $8 \%$ du produit national brut du Québec. Déjà considérables, les dommages causés à ce type d'environnement peuvent toutefois le devenir davantage, car avec la pollution des lacs, des rivières, c'est toute la chaîne alimentaire qui risque de se contaminer. Outre ces menaces à la santé publique, les pluies acides causent chaque année des dommages matériels de plus en plus importants, particulièrement en érodant des édifices, des statues et des monuments de pierres.

Une autre cause de dégradation de l'environnement réside dans 1'utilisation des pesticides. Au Québec, $40 \%$ des ventes de produits antiparasitaires sont constituées d'insecticides dangereux, lesquels sont

2. On appelle «pluies acides», les précipitations dont le taux d'acidité est inférieur à un ph 5,6 . Les principales sources canadiennes proviennent des dégagements d'oxyde d'azote des véhicules automobiles, de la thermoélectricité et des émanations d'oxyde de soufre résultant de la fusion des métaux non ferreux. Le Temps perdu, cri de ralliement contre les pluies acides, Rapport du sous-comité sur les pluies acides, Ottawa, juin 1984. Voir aussi Maryse Grandbois, «Le contrôle juridique des précipitations acides», les Cahiers de droit, vol. 26, $n^{\circ} 3$, septembre 1985, p. 592 ss.

3. Évaluations des connaissances sur le transport à distance des polluants atmosphériques et sur les dépôts acides, comité fédéral provincial de coordination de la recherche et de la surveillance, Ottawa, août 1986, $19 \mathrm{p}$.

4. Association québécoise de lutte contre les pluies acides, "Qu'est-il arrivé aux érables du Québec?», Bulletin Media, 1984, vol. 1, n`2, p. 8-9.

5. Il n'existe pas d'étude canadienne ou québécoise comptabilisant le coût de l'ensemble des dommages causés par les pluies acides. Toutefois, une étude américaine, commandée par le Environmental Protection Agency, permet d'évaluer l'ampleur de ces coûts. Elle établit à cinq milliards de dollars le coût des dommages causés à la nature et aux ouvrages artificiels, par les précipitations acides, dans le «Tiers Est» des États-Unis (Université de Wyoming, 1980). Environnement Canada, l'Odyssée des pluies acides, Ottawa, 1984, p. 3. 
responsables de 422 intoxications pour la seule année 19796. En mai 1984, le Département de santé communautaire de Rimouski constate un taux très élevé du syndrôme hémolytique et urémique chez les enfants habitant près des forêts traitées au fénitrothion?. En juin 1986, une vingtaine d'élèves sont incommodés par une vaporisation d'insecticide sur les arbres d'une propriété voisine d'une école primaire de Pierrefonds 8 .

Depuis quelques années, les scientifiques s'inquiètent de la diminution des ruchers et des insectes pollinisateurs. Diminution qu'ils attribuent à l'utilisation de pesticides dans le traitement du maïs-grain'. Les insectes pollinisateurs étant les premiers détruits par cette pollution, on peut facilement en estimer les conséquences, quand on sait qu'environ $15 \%$ de l'alimentation dépend de la pollinisation par les insectes 10 .

Aux problèmes environnementaux que créent aujourd'hui les précipitations acides et l'utilisation des pesticides, il faut ajouter les accidents provoqués par les déchets dangereux, si l'on veut obtenir un portrait général des dommages les plus importants. En ce domaine, les criminalités sont nombreuses et le plus souvent visibles.

On sait que le transport et le traitement des déchets dangereux exige la coopération de tous les intervenants, pour assurer la sécurité publique. Or, ces dernières années, les accidents se sont multipliés. En 1978, la Compagnie américaine de fer et métaux, chargée de vidanger des réservoirs d'hydrocarbures à Ville Lasalle, déverse le contenu des réservoirs dans une tranchée, plutôt que de transporter et traiter les

6. Le malathion, le diazinon et le chlordane. Les Pesticides: nécessaires et dangereux (Cahier 1), les Pesticides et l' environnement, ministère de l'Environnement du Québec-TELUQ, 1983, pp. 19-20.

7. «Une grave maladie infantile due à l'épandage des pesticides», la Presse, 2 mai 1984.

8. Il s'agit d'une vaporisation d'Orthene sur les arbres d'une propriété voisine d'une école primaire de Pierrefonds. "Élèves intoxiqués par de l'insecticide», la Presse, 4 juin 1986.

9. Pion, Suzanne, D. De Oliveira, A. Bélanger et C. Ritchot, «Traitements insecticides et activité de l'abeille domestique sur le maïs-grain au Québec», Symposium international sur la pollinisation, 27-30 septembre 1983, éd. INRA Pub., 1984 (les Colloques de l'INRA, $\mathrm{n}^{\circ} 21$ ).

10. Au Québec, pour chaque dollar que l'apiculteur obtient de son miel, il revient $12 \$$ en bénéfice à l'agriculture. De Oliveira, «Importance économique de la pollinisation par les insectes», Annales de la société entomologique du Québec, vol. 28, 1983, pp. 40 à 50 . 
hydrocarbures ${ }^{11}$. De 1978 à 1980, 11\% des camions-remorques accidentés sur les routes canadiennes transportent des déchets dangereux ${ }^{12}$. En 1980, le rapport de la Commission Grange révèle que $27 \%$ des déchets dangereux transportés par train au Canada enfreignent les prescriptions de sécurité de la Loi sur les chemins de fer ${ }^{13}$.

La plupart des atteintes à l'environnement demeurent impunies, même si les infractions sont souvent visibles. Aux yeux de l'État, la recherche de conformité prime sur la répression. Dans certains cas, la conclusion d'ententes et la fixation d'échéances, même lointaines, entre le ministère de l'Environnement et les entreprises, met en échec toutes les poursuites, tant pénales qu'administratives et civiles. C'est le cas de la Loi sur la qualité de l'environnement du Québec, qui interdit les poursuites pénales contre les entreprises ayant soumis un programme d'assainissement, lorsque ce programme a été approuvé par le sous-ministre et que l'entreprise en respecte toutes les exigences ${ }^{14}$. Quatre cents entreprises ont conclu de telles ententes, au Québec15.

Le droit pénal de l'environnement n'en existe pas moins. Malgré son application toute relative, il contient même davantage d'infractions que le droit pénal général. Dans cet article, nous questionnons ce droit pénal de l'environnement. Nous en cernerons d'abord les contours (première partie) pour tenter d'expliciter les facteurs d'impunité des infractions relatives à l'environnement (deuxième partie).

\section{PREMIÈRE PARTIE: LE DROIT PÉNAL STATUTAIRE}

Le développement récent du droit pénal de l'environnement montre qu'il se constitue parallèlement au Code criminel. Certaines infractions du Code criminel peuvent d'ailleurs être utilisées dans la lutte contre les pollutions: la négligence criminelle, la nuisance

11. Le procureur général de la province de Québec c. La compagnies américaine de fer et métaux et André Leduc, non publié, Cour des sessions de la paix, Montréal, 11 février 1983.

12. Vombert, E.M., «The Transportation of Dangerous Goods», 1983 Alberta Law Review 489.

13. La Loi sur les chemins de fer sanctionne ces infractions par une faible amende de 500 \$. Le rapport Grange souligne qu'on n'a pas intenté de poursuites depuis 1967. J. Grange, Report on the Mississauga Railway Accident Inquiry, Ottawa, 1980, p. 19 ss.

14. Loi sur la qualité de l'environnement, L.R.Q. c. Q-2, art. 116.2.

15. Giroux, Lorne, «Le droit québecois de l'environnement et l'équilibre des divers intérêts», Communication présentée à la Ve Conférence internationale de droit constitutionnel, Université Laval, 3 octobre 1987. 
publique, le méfait, etc. ${ }^{16}$. Mais l'État porte rarement de telles accusations et le Code criminel demeure d'une utilité limitée pour dissuader les pollueurs. Confrontée à ce constat, la Commission de réforme du droit du Canada, suivant en cela un courant de plus en plus répandu 17 n'en propose pas moins d'ajouter une nouvelle infraction au Code criminel, une infraction spécifique, dénommée crime contre l'environnement ${ }^{18}$.

Est-ce à dire que le Code criminel peut et doit prendre le relais du droit pénal statutaire de l'environnement? Ce droit, composé de dispositions à caractère parfois très technique, s'est à ce jour toujours vu accorder un rôle secondaire. Selon certains auteurs, il n'est pas perçu, dans l'ensemble de la population, comme un véritable droit pénal, «manifestant la réprobation que le corps social attache à certains comportements», mais plutôt comme une sorte de discipline exigée par la vie en société ${ }^{19}$. D'où son efficacité relative et sa dévaluation par rapport au Code criminel.

Outre ces facteurs exogènes, le droit pénal de l'environnement souffre par ailleurs d'une absence de concision et de précision. Complexe et dispersé, le droit pénal de l'environnement se caractérise par la multiplicité des infractions (A) et la disparité des sanctions (B).

\section{A. LA MULTIPLICITÉ DES INFRACTIONS}

Le droit pénal de l'environnement n'est pas une brancheautonome du droit, mais, si l'on peut dire, une des voies d'approche du droit de l'environnement. En principe, il vient renforcer et sanctionner le régime réglementaire et législatif général, par la mise en œuvre de divers moyens de répression.

Le droit de l'environnement prend spécialement en compte la relation dynamique qui s'établit entre les personnes et leur milieu; il a

16. Art. 202, 176 et 387 C. Cri. D'autres infractions pourraient être utilisées: possession de substances dangereuses (art. 77 à 79) ou de substances volatiles malfaisantes (art. 174), troubler la paix (art. 171), infractions contre les animaux (art. 400 à 403). Commission de reforme du droit du Canada, les Crimes contre l'environnement, Ottawa, 1985, pp. 59 à 69.

17. Résolution du Conseil européen de l'environnement (1977), proposant aux pays européens d'inclure au code penal «une ou plusieurs incriminations de pollution, de nuisance, de destruction, de dégradation ou autres atteintes à la nature. De telles infractions font notamment partie du droit pénal général des États-Unis, de l'Allemagne, de l'Espagne et du Japon.

18. Les Crimes Contre l'environnement, précité, p. 77.

19. Robert, Jacques-Henri et Martine Rémond-Guilloud, Droit pénal de l'environ nement, Paris, Éditions Masson, 1983, p. 23. 
pour objet la lutte contre les pollutions, la protection des équilibres génétiques, la conservation de la nature et des ressources naturelles ${ }^{20}$. Le droit pénal de l'environnement vient renforcer cet édifice normatif, pour en sanctionner l'application. Et ce, en droit provincial comme en droit fédéral.

Le droit fédéral de l'environnement a connu un développement très rapide depuis le début des années 1970 . À cette époque, il concernait surtout la conservation de la nature. Depuis, son champ d'application s'est considérablement élargi. Aujourd'hui, les principales lois fédérales en ce domaine traitent de la pollution de l'air et de l'eau ${ }^{21}$, de la pollution maritime ${ }^{22}$, du contrôle des pesticides ${ }^{23}$, de l'établissement des parcs nationaux et de la protection des oiseaux migrateurs ${ }^{24}$, du transport des marchandises dangereuses ${ }^{25}$ et de l'énergie atomique ${ }^{26}$. Chacune de ces lois met en œuvre son propre régime pénal et il n'existe aucun mécanisme de coordination entre elles. Une recherche effectuée en 1985 au Département des sciences juridiques de l'Université du Québec à Montréal, a permis de recenser 288 infractions différentes dans quatorze lois fédérales sur l'environnement et leurs règlements d'application ${ }^{27}$.

D'application plus directe, le droit québécois de l'environnement a lui aussi connu une évolution marquée depuis le début des années 1970. Il contient aujourd'hui un contingent important de lois et règlements et partant, d'infractions. Ces lois québécoises portent sur la qualité de l'environnement ${ }^{28}$, l'établissement de parcs et réserves ${ }^{29}$,

20. Pereira Reis, Joâo, Contributos para uma teoria do direito do ambiente, Secrétaria de estado do ambiente e dos recursos naturais, Lisbonne, 1987.

21. Loi sur la lutte contre la pollution atmosphérique, S.C. 1970-71-72 c. 47; Loi sur les ressources en eau du Canada, c. 5 (1 ${ }^{\mathrm{er}}$ suppl.)

22. Loi sur l'immersion des déchets en mer, S.C. 1974-75-76, c. 55; Loi sur la prévention de la pollution des eaux arctiques, S.R.C. $1970 \mathrm{l}^{\mathrm{er}}$ suppl, c. 2; Loi sur les pêcheries, S.R.C. 1970 c. F-14; Loi sur la marine marchande, S.R.C. 1970 S-9.

23. Loi sur les produits antiparasitaires, S.R.C. 1970 c. P-10; Loi sur les contaminants de l' environnement, S.C. 1974-75-76 c. 72.

24. Loi sur les parcs nationaux, S.R.C. $1970 \mathrm{c}$. N-13; Loi sur la faune du Canada, S.C. 1973-74 c. 21; Loi sur la convention concernant les oiseaux migrateurs, S.R.C. 1970 c. M-12.

25. Loi sur le transport des marchandises dangereuses, S.C. 1980-81-82-83 c. 36.

26. Loi sur le contrôle de l'énergie atomique, S.R.C. 1970 c. A-19.

27. Grandbois, Maryse (dir.), Recherche exploratoire en droit pénal fédéral de l'environnement. Recherche effectuée par Jean-Michel Montbriand, février-mars 1985.

28. Loi sur la qualité de l'environnement, L.R.Q. c. Q-2. R-26.

29. Loi sur les parcs, L.R.Q. c. P-9; Loi sur les réserves écologiques, L.R.Q. c 
l'exploitation contrôlée des ressources naturelles ${ }^{30}$, l'usage des pesticides $^{31}$ et la protection du patrimoine, naturel et bâti ${ }^{32}$. Chacune de ces lois met aussi en œuvre son propre régime pénal, ce qui n'est pas sans générer des cumuls d'infractions, les droits pénaux fédéral et provincial s'appliquant en même temps, sur le même territoire. Cela ne garantit pas pour autant la répression des infractions.

Les dispositions pénales sanctionnant les infractions au droit de l'environnement sont le plus souvent regroupées à la fin des textes de loi et de règlement. Elles sont rédigées en termes généraux et réfèrent presque toujours à d'autres articles de la même loi aux fins d'expliciter le contenu matériel de l'infraction. C'est l'ensemble de ces renvois et de ces dispositions pénales qui nous permettent de dresser une brève typologie des infractions.

De manière générale, les lois fédérales et québécoises sur l'environnement sanctionnent quatre catégories de comportement fautif, que l'on peut classer comme suit: le défaut d'autorisation administrative, le non-respect des normes réglementaires, le rejet de substances nuisibles dans l'environnement, le refus d'obtempérer à une demande administrative. Il va sans dire que chaque catégorie regroupe des infractions très différentes quant à leur gravité ou leurs conséquences. Ces catégories ne sont pas, non plus, équivalentes entre elles; tout au plus nous permettent-elles de présenter une vue d'ensemble du droit pénal de l'environnement, dont nous donnons ici un aperçu très sommaire.

\section{LE DÉFAUT D'AUTORISATION ADMINISTRATIVE}

Plusieurs lois sur l'environnement prescrivent l'obtention de certificats ou de permis d'opération, avant d'autoriser la poursuite de certaines activités. Le défaut d'obtention de ces certificats et/ou permis d'opération constitue une infraction; de même que le non-respect des conditions du permis.

La Loi sur la qualité de l' environnement du Québec exige l'obtention d'un certificat d'autorisation auprès du sous-ministre, avant toute action susceptible de modifier la qualité de l'environnement ${ }^{33}$;

30. Loi sur la conservation et la mise en valeur de la faune, L.R.Q. c. 61.1; Loi sur les forêts, L.Q. 1986 c. 108; Loi sur les mines, L.Q. 1986 c. 64.

31. Loi sur les pesticides, L.Q. 1987 c. 29.

32. Loi sur les biens culturels, L.R.Q. c. B-4.

33. Loi sur la qualité de l' environnement, art. 22 et 106. 
La Loi sur les biens culturels du Québec exige l'autorisation du ministère des Affaires culturelles pour l'aliénation d'un bien culturel classé34;

La Loi fédérale sur l'immersion des déchets en mer prescrit l'obtention d'un permis avant toute immersion de déchets en mer 35 ;

La Loi sur le contrôle de l'énergie atomique exige l'obtention de permis d'exploitation pour les établissements nucléaires ${ }^{36}$;

La Loi sur la faune du Canada et le Règlement sur les réserves de faune exigent l'obtention de permis pour la chasse et la pêche dans les réserves de faune ${ }^{37}$.

\section{LE NON-RESPECT DES NORMES RÉGLEMENTAIRES}

Certaines lois sur l'environnement délèguent au Conseil des ministres un pouvoir réglementaire très étendu. C'est le cas par exemple de la loi québécoise sur la qualité de l'environnement et de la loi fédérale sur la prévention de la pollution des eaux arctiques. Des règlements déterminent alors les quantités maximales pour l'émission ou le rejet de contaminants. Le non-respect de ces normes constitue une infraction.

Le règlement québécois sur la qualité de l'atmosphère prescrit des normes de concentration moyenne maximale pour certains contaminants, tels l'anydride sulfureux, le dioxyde d'azote et le monoxyde de carbone ${ }^{38 ;}$

Le règlement québécois sur les fabriques de pâtes et papiers impose des normes pour les émissions d'anhydride sulfureux et de matières particulières ${ }^{39}$;

La Loi sur la lutte contre la pollution atmosphérique permet au gouvernement fédéral de réglementer la teneur en plomb de

34. Loi sur les biens culturels, art. 32 et 56.

35. Loi sur l' immersion de déchets en mer, art. 4.

36. Loi sur le contrôle de l'énergie atomique, art. 19(1).

37. Règlement sur les réserves de faune, C.R.C. c. 1609, art. 3(1) a).

38. Règlement sur la qualité de l'atmosphère, R.R.Q. 1981, c. Q-2 r 20; Loi sur la qualité de l'environnement, art. 20 et 106.

39. Règlement sur les fabriques de pates et papiers, R.R.Q. 1981, c. Q-2 r 12; Loi sur la qualité de l'environnement, art. 20 et 106. 
l'essence ${ }^{40}$, et d'imposer des normes de dégagement pour les mines et les usines d'extraction d'amiante ${ }^{41}$;

Le règlement fédéral sur le transport des marchandises dangereuses prescrit des normes de sécurité pour le transport de certaines catégories de produit'42;

Le règlement sur la prévention de la pollution des eaux arctiques prescrit des normes pour le dépôt des déchets industriels ${ }^{43}$.

\section{REJET DE SUBSTANCES NUISIBLES DANS L'ENVIRONNEMENT}

D'autres lois sur l'environnement interdisent le rejet de substances nuisibles et dangereuses dans l'environnement. Le rejet de ces substances constitue une infraction.

La Loi sur la qualité de l'environnement du Québec interdit le rejet de tout contaminant susceptible de porter atteinte à la vie, à la santé, à la sécurité, au bien-être et au confort de l'être humain, de causer des dommages ou de porter autrement préjudice à la qualité du sol, à la végétation, à la faune ou aux biens ${ }^{44}$;

La Loi sur les pêcheries du Canada interdit le rejet de substances nuisibles ou délétères dans les eaux où se fait la pêche ${ }^{45}$;

Le règlement fédéral sur la convention concernant les oiseaux migrateurs interdit les dépôts de substances nocives dans les eaux ou les régions fréquentées par les oiseaux migrateurs ${ }^{46}$.

40. Règlement sur l'essence au plomb, C.R.C. c. 409, Loi sur la lutte contre la pollution atmosphérique, art 22.

41. Règlement sur les normes de dégagement pour les mines et les usines d'extraction d'amiante, C.R.C. c. 405; Loi sur la lutte contre la pollution atmosphérique, art. 22.

42. Règlement sur le transport des marchandises dangereuses, C.P. 1985-147, 17 janvier 1985; Dors/85-77, GC partie II 1985, p. 393; Loi sur le transport des marchandises dangereuses, art. 4.

43. Règlement sur la prévention de la pollution des eaux arctiques, C.R.C. c. 354; Loi sur la prévention de la pollution des eaux arctiques, art. 4,9.

44. Loi sur la qualité de l' environnement, art. 20 et 106.

45. Loi sur les pêcheries, art. 33(1).

46. Règlement sur les oiseaux migrateurs, C.R.C. 1978 c. 1035, art. 35(1) 


\section{LE REFUS D'OBTEMPÉRER À UNE DEMANDE ADMINISTRATIVE}

Plusieurs lois sur l'environnement confèrent d'importants pouvoirs administratifs au ministre ou au sous-ministre. Dans certains cas, le refus de se conformer à une demande émanant de l'autorité administrative constitue une infraction.

En vertu de la Loi sur la qualité de l'environnement, le ministre peut rendre, à l'égard d'une personne exploitant un lieu d'élimination, d'entreposage ou une usine de traitement des déchets, les ordonnances qu'il juge appropriées relativement à la qualité du service, aux rapports à faire, aux modalités d'exploitation ${ }^{47}$;

En vertu de la Loi sur la lutte contre la pollution atmosphérique, le ministre fédéral de l'environnement peut exiger des échantillons des matières dégagées ou utilisées par une entreprise ${ }^{48}$; le ministre peut exiger des preuves de solvabilité, selon la Loi sur la prévention de la pollution des eaux arctiques ${ }^{49}$. Il peut demander des renseignements et exiger des expériences, en vertu des dispositions de la Loi sur les contaminants de l'environnement ${ }^{50}$.

Ce bref aperçu des catégories d'infractions du droit pénal de l'environnement laisse entrevoir assez clairement la multiplicité des dispositions répressives et leur caractère disparate. Quant aux sanctions prévues par ces lois, elles se révèlent elles aussi inégales dans leur formulation même.

\section{B. LA DISPARITÉ DES SANCTIONS}

Le droit pénal de l'environnement se construit autour d'une morale, selon laquelle la pollution constitue une atteinte au bon fonctionnement du corps social. À ce titre, la pollution doit être réprimée et punie. Aussi, les infractions à l'environnement sont-elles assorties de sanctions: confiscation de certains biens, peines d'emprisonnement, amendes plus ou moins importantes, ordonnances et/ou injonctions diverses, dont la fonction doit être de dissuader les pollueurs. Ces peines varient selon les lois, les infractions et parfois même selon les modes de poursuite ${ }^{51}$.

47. Loi sur la qualité de l' environnement, art. 57 et 107.

48. Loi sur la lutte contre la pollution atmosphérique, art. 6, 34(1).

49. Loi sur la prévention de la pollution des eaux arctiques, art. 5(1).

50. Loi sur les contaminants de l' environnement, art. 3 et 4.

51. Les lois fédérales prévoient souvent deux types de sanction, selon la poursuite intentée par le procureur général. La Loi sur la protection des pêcheries côtières, par 
L'écart entre ces diverses sanctions peut se révéler très grand, pour des offenses de gravité similaire. De fait, à la lecture des textes de lois sur l'environnement, le droit pénal semble difficilement prévisible et potentiellement très irrégulier dans son application. Nous en retraçons ici quelques exemples, en présentant l'inventaire des sanctions possibles pour des problèmes types en droit de l'environnement: la pollution atmosphérique et la pollution maritime.

\section{POLLUTION ATMOSPHÉRIQUE}

L'émission de polluants, au-delà des normes autorisées - infraction industrielle fréquente - peut faire l'objet de sanctions pénales diverses telles l'imposition d'amendes plus ou moins importantes et/ou de peines d'emprisonnement.

Ainsi, l'émission d'oxyde de soufre au-delà des normes autorisées, rend les personnes enfreignant le Règlement sur la qualité de l'atmosphère passibles d'une peine d'emprisonnement ${ }^{52}$, mais la même infraction au Règlement sur les fabriques de pâtes et papiers n'est sanctionnée que par l'amende seulement ${ }^{53}$. Par ailleurs, si cette même infraction est commise en territoire fédéral, l'amende peut atteindre 200000 \$, et être accompagnée d'une injonction enjoignant de cesser toute activité pouvant entraîner la récidive ${ }^{54}$. D'autre part, l'émission d'un polluant réglementé en vertu de la loi fédérale sur les contaminants ${ }^{55}$ peut entrainer l'imposition d'une peine d'emprisonnement ${ }^{56}$ alors qu'une poursuite en droit québécois, pour la même infraction, n'est passible que de la peine générale prévue à la Loi sur la qualité de l'environnement, soit l'amende seulement ${ }^{57}$.

exemple, rend les contrevenants passibles d'une amende maximale de $5000 \$ \mathrm{et} / \mathrm{ou} \mathrm{d}$ 'un emprisonnement d'au plus trois mois, sur déclaration sommaire de culpabilité. Après une déclaration de culpabilité sur acte d'accusation, l'amende peut atteindre $25000 \$$ et l'emprisonnement deux ans. Loi sur la protection des pêcheries côtières, S.R.C. $1970 \mathrm{c}$. C-21, art. 8.

52. Règlement sur la qualité de l'atmosphère, art. 96.4, 96.5, 96.6.

53. Règlement sur les fabriques de pâtes et papiers, art. 52.

54. Loi sur la lutte contre la pollution atmosphérique, art. 33(1), 35.

55. Loi sur les contaminants de l' environnement, art. 8(5) b).

56. Loi sur la qualité de l' environnement, art. 106 et 107.

57. Il s'agit d'un domaine de juridiction fédérale. 
TABLEAU 1

Pollution atmosphérique

Sanctions

\section{Lois québécoises}

Loi sur la qualité de l' environnement

$\begin{array}{llrlr}\text { personnes physiques: } & \text { amendes } & 200 \$ & \text { à } & 5000 \$ \\ \text { récidive: } & & 400 \$ & \text { à } & 10000 \$ \\ \text { corporations: } & \text { amendes } & 600 \$ & \text { à } & 30000 \$ \\ \text { récidive: } & & 1200 \$ & \text { à } & 60000 \$\end{array}$

(pour chaque jour durant lequel l'infraction s'est poursuivie)

\section{Règlement sur la qualité de l'atmosphère}

$\begin{array}{llrrr}\text { personnes physiques: } & \text { amendes } & 100 \$ & \text { à } & 5000 \$ \\ \quad \text { récidive: } & & 200 \$ & \text { à } & 25000 \$ \\ \begin{array}{l}\text { emprisonnement: } \\ \text { corporations: }\end{array} & 1 \text { à } 6 \text { mois } & & & \\ \quad \text { récidive: } & \text { amendes } & 500 \$ & \text { à } & 50000 \$ \\ & & 2000 \$ \text { à } & 100000 \$\end{array}$

Règlement sur les fabriques de pâtes et papiers

$\begin{array}{llrll}\text { personnes physiques: } & \text { amendes } & 200 \$ & \text { à } & 10000 \$ \\ \text { récidive: } & & 400 \$ & \text { à } & 25000 \$ \\ \text { corporations: } & \text { amendes } & 600 \$ & \text { à } & 30000 \$ \\ \text { récidive: } & & 1200 \$ & \text { à } & 60000 \$\end{array}$

\section{Lois fédérales}

Loi sur la lutte contre la pollution atmosphérique

amende maximale: $200000 \$$

injonction

Loi sur les contaminants de l'environnement

déclaration sommaire de culpabilité: amende maximale: $5000 \$$

acte d'accusation: emprisonnement: 2 ans

\section{POLLUTION MARITIME}

Les lois fédérales relatives à la pollution maritime présentent elles aussi les mêmes disparités. Pour des infractions de même nature, tel le rejet de polluants dans la mer, les sanctions varient selon l'inculpation et selon les lois. En ce domaine, nous sommes en présence de quatre lois. Deux sont héritées du XIXe siècle et ont été modifiées au cours des années pour inclure des dispositions visant à assurer une meilleure protection de l'environnement marin. 
TABLEAU 2

\section{Pollution maritime}

Sanctions

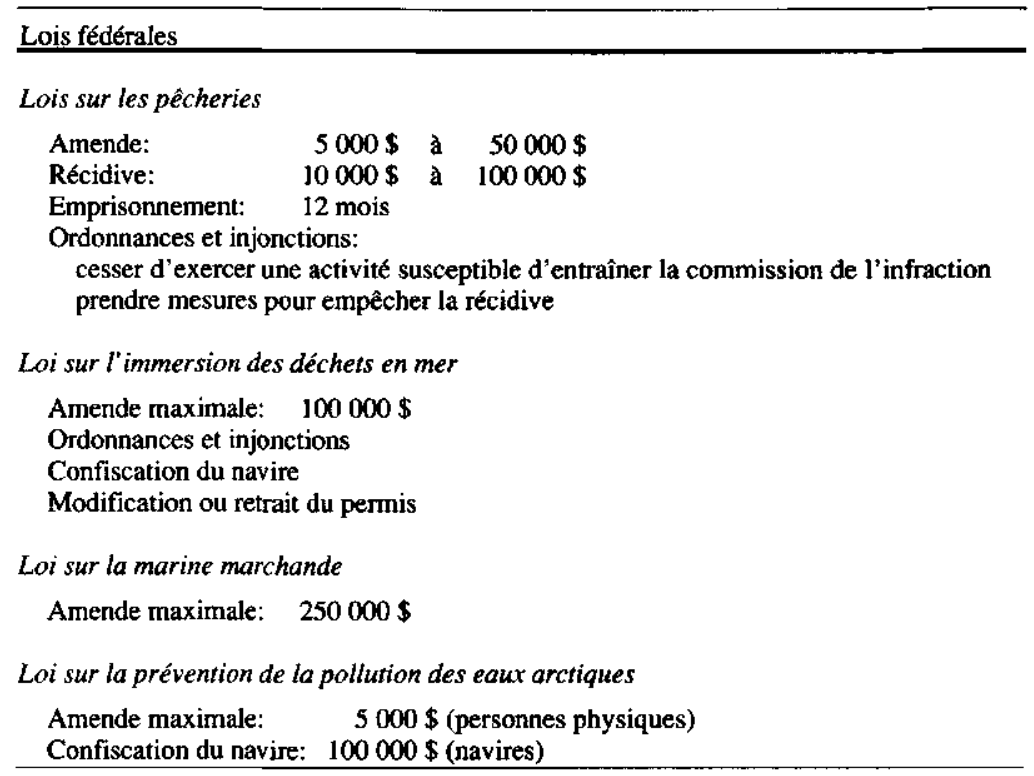

Selon l'une de ces deux lois, la Loi sur les pêcheries, l'infraction consistant à rejeter des polluants à la mer peut se voir sanctionnée d'une amende relativement faible (5000\$) ou importante (50 000\$), selon l'inculpation ${ }^{58}$. L'amende peut être accompagnée d'une injonction ou d'une ordonnance enjoignant de prendre des mesures pour éviter la récidive. Pour la même infraction, la Loi sur la marine marchande impose une amende maximale de $250000 \$ 59$.

Les deux autres lois datent des années 1970 et visent spécifiquement le contrôle de deux types de pollution: la pollution des mers par les déchets et la pollution plus spécifique des eaux et des côtes arctiques, milieu particulièrement fragile. Selon ces lois, la même infraction consistant à rejeter des déchets à la mer sans permis peut à la fois être sanctionnée de manière plus rigoureuse et entraîner la confiscation du navire.

58. Loi sur les pêcheries, art. 61(1), art. 30, 33(2).

59. Loi sur la marine marchande, art. 728 et 752 . 
En vertu de la Loi sur l'immersion des déchets en mer, l'amende imposée peut atteindre $100000 \$$ et peut être assortie d'injonction, d'ordonnance et de la confiscation du navire, ainsi que du retrait ou de la modification du permis ${ }^{60}$. La Loi sur la prévention de la pollution des eaux arctiques $^{61}$ sanctionne les infractions des personnes physiques par une amende maximale de $5000 \$$, et les infractions des navires par une amende maximale de $100000 \$$. Le juge peut aussi ordonner la confiscation du navire.

\section{LES AMENDES}

Toutes les lois sur l'environnement sanctionnent les infractions par l'amende (tableau 3) ${ }^{62}$. Celles-ci peuvent varier selon la gravité de l'infraction, les conséquences possibles, l'importance que l'État accorde à ce type d'infraction. Dans certains cas, on le constate à la lecture du tableau 3, les amendes peuvent être très élevées. Le droit fédéral, par exemple, prescrit l'imposition d'amendes maximales plus élevées que le droit provincial, sauf en ce qui concerne les infractions relatives à la loi ou au règlement sur les parcs nationaux. Ces dernières sont sanctionnées par une amende maximale de $500 \$$.

Une aussi faible amende se révèle peu dissuasive, en particulier en ce qui concerne le braconnage d'espèces rares. Comme les oiseaux et les animaux protégés peuvent se vendre jusqu'à 50000 \$ sur le marché des collectionneurs, les réseaux de braconniers, régulièrement découverts et démantelés, se recréent aussitôt. Tels par exemple ces réseaux mis au jour en 1984, et faisant le trafic de faucons pélerins entre les États-Unis et le Canada ${ }^{63}$ ou «l'exportation» de mouflons des Rocheuses du parc national de Banff64.

En droit québécois, la Loi sur la qualité de l'environnement impose aux corporations une échelle d'amendes plus élevées, pouvant être modulée en fonction de leur capacité de payer. Cela n'en fait pas pour autant une loi particulièrement dissuasive pour les grandes entreprises.

60. Loi sur l' immersion des déchets en mer, art. 13(1) (2) et (3).

61. Loi sur la prévention de la pollution des eaux arctiques, ant. 18(1), (9).

62. Loi sur les biens culturels, art. 58.1; Loi sur les parcs, art. 11,11.1, 11.2; Loi sur la conservation et la mise en valeur de la faune, art. 165 à 171.1; Loi sur les contaminants de l'environnement, art. 8(5); Loi sur les produits antiparasitaires, art. 10(1); Loi sur le transport des marchandises dangereuses, art. 6(1); Loi sur le contrôle de l'énergie atomique, art. 19(1); Loi sur les parcs nationaux, art. 8(1) a).

63. «Trafic de faucons", le Devoir, 30 juin 1984.

64. «Alberta draws a bead on big-game poachers», Globe and Mail, 27 décembre 1983. 
TABLEAU 3

Amendes

Lois québécoises

Loi sur la qualité de l' environnement

$\begin{array}{lllr}\text { personnes physiques: } & 100 \$ & \text { à } & 5000 \$ \\ \text { récidive: } & 200 \$ & \text { à } & 10000 \$ \\ \text { corporations: } & 300 \$ \text { à } & 30000 \$ \\ \text { récidive: } & 600 \$ \text { à } & 60000 \$\end{array}$

Loi sur les réserves écologiques

récidive:

$25 \$$ à $300 \$$

$300 \$$ à $1000 \$$

Loi sur la conservation et la mise en valeur de la faune

récidive:

$200 \$$ à $1500 \$$

$600 \$$ a $13500 \$$

Loi sur les parcs

$25 \$$ à $5000 \$$

Loi sur les biens culturels

$500 \$$ à $50000 \$$

\begin{tabular}{ll}
\hline Lois fédérales & \\
\hline Loi sur les contaminants de l'environnement* & $100000 \$$ (max.) \\
& \\
Loi sur les produits antiparasitaires* & $100000 \$$ (max.) \\
& \\
Loi sur le transport des marchandises dangereuses* & $50000 \$$ (max.) \\
récidive: & $10000 \$$ (max.) \\
Loi sur le contrôle de l'énergie atomique* & $5000 \$$ \\
Déclaration sommaire de culpabilité: & $10000 \$$ \\
Acte d'accusation: & \\
Loi sur les parcs nationaux* & $500 \$$ (max.) \\
\hline
\end{tabular}

* Le contrevenant peut aussi être condamné à une peine d'emprisonnement.

Aussi les amendes sont-elles parfois perçues comme «un simple droit de permis d'exercer des activités illégales» 65 . D'autant plus qu'en droit de l'environnement, l'amende maximale n'est que très rarement imposée au contrevenant.

65. Swaigen, John et Gail Bunt, la Détermination de la peine en droit de l'environnement, Commission de réforme du droit du Canada, 1985, p. 17. 
Certaines lois prévoient d'autres sanctions en sus des amendes, en particulier en droit fédéral: confiscation des biens, suspension ou révocation des permis, emprisonnement, ordonnances de faire ou de cesser de faire, injonctions... Mais ces sanctions sont, elles aussi, rarement imposées.

De fait, le droit pénal de l'environnement ne manque ni d'infractions ni de sanctions. Bien qu'éclatés et disparates, les mécanismes de répression des crimes contre l'environnement permettent même de moduler les sanctions en fonction de la gravité des infractions et de la capacité de payer des entreprises. Nous devons cependant constater que ce droit pénal ne remplit pas les fonctions sociales de répression et de dissuasion qu'il prétend exercer. Pour cerner l'ampleur de ce problème, nous questionnerons maintenant la mise en ceuvre de ce droit par l'administration publique et les tribunaux.

\section{DEUXIÈME PARTIE: LES FACTEURS D'IMPUNITÉ}

Prosecution, let alone jail, is seen as an alternative only after such negotiations are deemed to have failed, and when "warnings" are not heeded. Do people guilty of criminal negligence, robbery or attempted murder get a warning or continued warnings ${ }^{66}$ ?

Les crimes contre l'environnement ne differrent pas fondamentalement des crimes traditionnels contre la sécurité des personnes et des biens. Il s'agit, la plupart du temps, d'actes commis de propos délibéré, sans aucun souci des conséquences pour l'environnement ou la santé publique; d'actes justifiés par «l'impérieuse nécessité d'éviter toute augmentation des coûts de production» et motivés par le profit escompté de ces activités illégales. Ces crimes contre l'environnement sont souvent des actes répétés, presque habituels, comme la vidange de citernes, le dépôt de déchets toxiques ou l'émission de polluants audelà des normes autorisées.

Selon le directeur de la brigade criminelle de l'Environmental Protection Agency, dans les causes de droit pénal de l'environnement, les défendeurs ne peuvent présenter aucune défense; ils ne se sont pas conformés aux lois et règlements, disent-ils, parce que cela impliquait des dépenses trop importantes ${ }^{67}$.

L'administration publique canadienne et québécoise recourt très peu au droit pénal pour obtenir la conformité des activités industrielles

66. «Prosecuting Polluters - You've got to be in it to win», Editorial, Intervenor, vol. $12, \mathrm{n}^{\circ} 1$, janvier-février 1987.

67. Starr, Judson W., «Countering Environmental Crimes», 13 B.C. Envt' I Aff. Law Rev., p. 382. 
aux lois et règlements. Cette conformité est recherchée à l'extérieur des mécanismes pénaux, voire même à l'extérieur du droit (A).

Lorsque les négociations échouent, des poursuites peuvent être intentées, mais ces poursuites ne se terminent pas toujours par l'imposition de sanctions dissuasives 68 . En principe, les amendes imposées devraient, à tout le moins, être plus importantes que les gains illégalement obtenus ${ }^{69}$. Cependant, à cause de l'importance économique et sociale de certains pollueurs, les tribunaux hésitent encore fréquemment à imposer des amendes très élevées et des sanctions véritablement dissuasives (B).

\section{A. L'ADMINISTRATION DU DROIT DE L'ENVIRONNEMENT : LA RARETÉ DES POURSUITES}

Au Québec comme au Canada, de manière générale, il n'est pas interdit de polluer. Ce qui est interdit, c'est de dépasser une certaine limite de pollution. Or, cette limite s'avère très difficile à fixer, en raison des multiples intérêts en jeu. Rappelons que cette limite est déterminée en fonction de la capacité de renouvellement des ressources naturelles et de la capacité «d'assimilation maximale» de l'environnement, toutes choses sur lesquelles les avis divergent selon les intérêts.

C'est en raison de cette diversité d'avis et d'intérêts que l'État multiplie et justifie les conditions particulières qui permettent à certains pollueurs de continuer leurs opérations. Au mieux doivent-ils, la plupart du temps, respecter minimalement les règlements d'application des lois sur l'environnement.

Il est important de noter que l'inégalité de traitement découle de la notion même de gestion des pollutions. Selon ce principe de gestion, comme les pollutions ne peuvent pas être maintenues à leur niveau antérieur, il faut gérer l'ensemble des dépassements. Éventuellement, tous les pollueurs devront se conformer aux règlements. En attendant, l'État gestionnaire effectue des choix. Et ce sont les plus gros pollueurs et les plus importantes entreprises qu'il choisit de privilégier. C'est le cas, par exemple de la compagnie Mines Noranda, qui peut enfreindre le Règlement sur la qualité de l' atmosphère jusqu'en 199469a.

68. Swaigen, John et Gail Bunt, la Détermination de la peine en droit de l'environnement, précité, p. 8 ss.

69. Wilson, John D., «Re-thinking Penalties for Corporate Environmental Offenders: a View on the Law Reform Commission of Canada's Sentencing in Environment Cases», 1986 Mc Gill Law Journal, 31, p. 321.

69a. Ibid 
En fait, la situation n'est pas bien différente des années 1940 ou 1950. À cette époque et jusqu'en 1965, les poursuites civiles contre la compagnie Mines Noranda étaient interdites, en vertu d'une disposition législative ${ }^{70}$. Rappelons au passage que l'histoire du droit québécois est constellée de ces appuis presque inconditionnels de l'État à la grande entreprise ${ }^{71}$.

Cette situation n'est pas particulière au Québec et au Canada. Les sociétés multinationales produisent plus du tiers du produit mondial brut. À ce titre, elles exercent parfois un pouvoir réglementaire plus étendu que les États, en matière d'environnement ${ }^{72}$. Il faut souligner qu'en ce domaine les gouvernements canadiens se trouvent particulièrement vulnérables, étant donné le pourcentage élevé de capitaux étrangers dans l'économie nationale ${ }^{73}$.

Les gestionnaires disposent toutefois de plusieurs moyens d'action pour que les entreprises se conforment aux objectifs publics de protection de l'environnement. D'autant plus que certains de ces moyens sont extrêmement favorables aux entreprises. Il en va ainsi, par exemple des diverses mesures d'incitation financière : prêts, subventions, garanties d'emprunt, dégrèvements fiscaux. L'État absorbe ainsi $25 \%$ du coût du programme de modernisation des usines de pâtes et papiers. Les dégrèvements fiscaux aux entreprises totalisent 6,2 milliards de dollars au Canada pour la seule année $1983^{74}$.

Malgré les avantages qu'elles confèrent aux entreprises, ces mesures d'incitation financière ne sont pas toutes efficaces. Le programme d'amortissement accéléré de l'équipement antipollution, par exemple, qui coûte à lui seul plus de 100 millions de dollars par an au trésor public, se révèle, aux yeux de certains économistes, à la fois peu efficace et peu équitable. Dans l'administration de ce programme, l'État n'exige aucune preuve de qualité de l'équipement antipollution. Par ailleurs, l'amortissement accéléré a pour effet de transférer le coût

70. Loi des mines, S.R.Q. 1964 c. 89, art. 121, 122. Ces articles ont été abrogés par la Loi sur les mines, S.Q. 1965, c. 34, art. 248.

71. Voir par exemple, les lois ayant favorisé le développement de l'industrie de la pêche en Gaspésie. Maryse Grandbois, «Le développement des disparités régionales en Gaspésie", Revue de l'histoire de l'Amérique française, vol. 36 nº 4, mars 1987, pp. 483 à 506.

72. Schrecter, T.F., l'Élaboration des politiques en matière d'environnement, précité, p. 68.

73. Ibid., p. 79.

74. Commission de réforme de droit du Canada, Droit, objectifs publics et observation des normes, Document de travail n 51 , Ottawa, 1986, p. 51 ss. 
de la pollution des pollueurs aux non-pollueurs et bénéficie surtout aux plus grosses entreprises 75 .

Malgré ces constats, l'administration publique estime que l'ensemble des mesures d'incitation financière se révèle plus utile pour obtenir des entreprises la conformité aux prescriptions environnementales. Aussi les poursuites sont-elles peu nombreuses, alors que les ententes et les régimes particuliers se multiplient ${ }^{76}$.

L'approbation d'un programme particulier d'assainissement par le sous-ministre met les entreprises à l'abri des poursuites pénales, si les entreprises respectent les exigences et échéances du programme ${ }^{77}$. Dans tous les cas, ce type d'immunité permet aux entreprises de continuer leurs opérations polluantes pendant toute la durée du programme d'assainissement (deux à cinq ans), et ce, malgré la réglementation en vigueur. Comme nous l'avons déjà mentionné, environ 400 entreprises se sont prévalues ou se prévalent actuellement de ce programme.

Selon Lome Giroux, la signature de telles ententes tend à devenir «le seul mode de contrôle d'un secteur entier de l'activité économique» 78 . Ce qui, estime-t-il, risque d'institutionnaliser l'arbitraire administratif, d'autant plus que ces ententes individuelles d'assainissement s'ajoutent à des régimes particuliers qui dotent déjà l'administration publique de pouvoirs discrétionnaires. Et, Lorne Giroux de conclure : «le simple citoyen, poursuivi pour avoir déversé du sable en bordure de sa propriété riveraine, est en droit de s'interroger sur l'équité d'un système juridique en vertu duquel une entreprise qui opère depuis 1962 et qui déverse 300 tonnes métriques d'acide sulfurique par jour dans le fleuve Saint-Laurent n'a jamais fait l'objet d'une seule poursuite»79.

L'administration publique agit comme si, à long terme, les entreprises allaient de toutes façons se conformer volontairement aux règlements. Ou encore, comme si une innovation technologique

75. Allin, Joan, «The Tax Subsidy for Pollution Abatment Equipment», Canadian Taxation, été 1979, p. 47. Voir aussi Leonard Waverman, Fiscal Instruments and Pollution : An Evaluation of Canadian Legislation», 1970 Canadian Tax Journal, vol. XVIII, n 1 , pp. 505 à 513.

76. C.R.D.C., Droit, objectifs publics et normes, précité, p. 43.

77. Loi sur la qualité de l'environnement, art. 116.2.

78. Giroux, Lome, «Le droit québécois de la qualité de l'environnement et l'équilibre des divers interêts», Ve Conférence internationale de droit constitutionnel, Communication présentée le 30 octobre 1987, pp. 37-38.

79. Ibid, p. 38. 
pouvait tout à coup régler tous les problèmes de pollution. Ce qui fait que la plupart des infractions ne font pas l'objet de poursuites mais entraînent plutôt la renégociation d'ententes particulières et de délais ${ }^{80}$.

Lorsque la décision d'intenter une poursuite est prise, cette décision se fonde sur une série de facteurs tout aussi optimistes. Notamment, sur le comportement de l'entreprise, les efforts déployés pour corriger la situation, la probabilité qu'une autre autorité administrative exerce une action ${ }^{81}$. En cas de poursuite, on accorde autant d'attention à l'attitude des corporations qu'à leurs activités polluantes.

Selon Keith Hawkins, l'administration publique distingue quatre types de comportement qui atténuent ou aggravent la responsabilité des entreprises, selon qu'elles semblent ou non faire preuve de bonne volonté. C'est ainsi que l'on distingue les entreprises qui se conforment aux règlements; celles qui ne s'y conforment pas par incapacité financière ou technique; celles qui ne s'y conforment pas par négligence; et celles qui enfreignent volontairement les règlements, par malice 82 .

L'administration publique semble réserver le recours au droit pénal aux infractions de cette quatrième catégorie, les infractions odieuses, tel, par exemple, le déversement de déchets toxiques dans un cours d'eau.

Ce recours au droit pénal par l'administration publique s'exerce seulement après l'échec des négociations, lorsqu'il ne subsiste aucun espoir de voir cette entreprise se conformer aux lois et règlements ${ }^{83}$. Il en résulte que le recours au droit pénal ne sert pas à rencontrer le droit, à prévenir les infractions par crainte des sanctions, il sert plutôt $a$ posteriori, en dernier recours, à justifier les actions de l'administration, quand celle-ci considère que l'heure est venue de punir.

En droit québécois, toute personne peut formuler une plainte pénale et initier des poursuites, «sauf si la loi qui crée la contravention exige une autorisation spéciale ${ }^{84}$ ». Jusqu'en 1978, la Loi sur la qualité de l'environnement requérait l'autorisation du procureur général avant

80. Rankin, Murray et Peter Finkle, «The Enforcement of Environmental Law : Taking the Environment Seriously», U.B.C. Law Rev., 1983, vol. 17, 1, p. 42.

81. Droit, objectifs publics et normes, Commission de réforme du droit du Canada, précité, pp. 72-73.

82. Hawkins, Keith, Environment and Enforcement, Clarendon Press, Oxford, 1984, p. 112.

83. Jbid., p. 107.

84. Loi sur les poursuites sommaires, L.R.Q. c. P-15, art. 12(2). 
toute poursuite. Cette exigence n'a pas facilité le développement du droit pénal de l'environnement.

La suppression de cette autorisation préalable n'a pas contribué de façon significative à l'augmentation du nombre des poursuites. Et ce, en raison d'un problème de taille : l'accès à l'information. Certes, à l'heure actuelle, l'administration doit répondre aux demandes d'information, mais seulement dans la mesure où 1 'administration considère l'information disponible85. De plus, les demandes d'information sont elles-mêmes soumises à certaines restrictions de la Loi sur l'accès à l'information et la protection des renseignements personnels $s^{86}$. On se rappellera à ce propos que, récemment, le Devoir n'a pu obtenir copie complète de l'entente intervenue entre le ministère de l'Environnement du Québec et la compagnie Mines Noranda 87.

Comme on l'a dit plus haut, toute personne peut en principe formuler une plainte pénale, mais il faut reconnaître que c'est l'administration qui fait, applique et interprète le droit de l'environnement. C'est également l'administration qui commande la procédure, mène la recherche, la constatation et la poursuite des infractions.

Enfin, soulignons le fait que peu d'infractions au droit fédéral de l'environnement font l'objet de poursuites judiciaires en territoire québécois. Selon le Conseil consultatif canadien de l'environnement, la rareté de ces poursuites tient à des raisons politiques. L'administration fédérale hésite à intenter des poursuites pour des infractions commises dans l'est du Canada, pour éviter les conflits de juridiction fédérale-provinciale qui pourraient en résulter ${ }^{88}$. Cette préoccupation politique ne favorise pas l'application du droit pénal de l'environnement, en l'absence d'une coordination étroite entre les administrations des deux paliers de gouvernement.

\section{B. LES TRIBUNAUX : LA DIFFICULTÉ DES POURSUITES}

Si l'administration publique ne recourt pas davantage aux poursuites pénales, cela tient aussi en partie à la pauvreté du support technique qu'elle trouve auprès du système judiciaire, de même qu'aux longs délais et aux coûts élevés de la justice canadienne et québécoise. De

85. Loi sur la qualité de l'environnement, art. 118.4.

86. Ibid., al. 2.

87. «Québec cache des passages du contrat», le Devoir, vendredi 2 octobre 1987.

88. Giroux, Lorne, Rapport du Conseil consultatif canadien de l' environnement concernant l'application par Environnement Canada de la réglementation relevant de son ministère, C.G.C.E. - Environnement Canada, juin 1985, 7 p. 
plus, comme les autorités administratives tiennent à gagner leurs procès, «l'incertitude» du processus judiciaire et les exigences de preuve contribuent à freiner les poursuites 89 .

Le ministère de l'Environnement du Québec s'est doté en 1987 d'un service des enquêtes, à l'instar du ministère ontarien de l'Environnement. Rappelons qu'aux États-Unis, la création de l'Environmental Crimes Unit de l'Environmental Protection Agency, en 1982, a contribué considérablement à augmenter le nombre de poursuites et de condamnations des corporations, pour des infractions au droit de l'environnement. Composé principalement d'enquêteurs de la police criminelle, l'Environmental Crime Unit prépare les dossiers, intente des poursuites et réclame des sanctions sévères.

Les résultats ne se font pas attendre. De 1983 à 1986, l'Environment Crime Unit initie cent quatre-vingt poursuites. Cent trente d'entre elles se soldent par une condamnation. Le Service rapporte un total cumulatif de 1,5 millions de dollars d'amende et dix ans de prison pour l'ensemble de ces poursuites. Cinquante entreprises ont été poursuivies comme entités corporatives; les quatre-vingt autres procès ont levé le voile corporatif et mettaient en cause les officiers de la compagnie ${ }^{90}$.

Plus près de nous, en Ontario, deux jugements de 1986 ont condamné des présidents de compagnie à l'emprisonnement.

Le président de la compagnie Jetco Manufacturing Limited, $\mathbf{M}$. Keith Alexander, déjà condamné à 97950 \$ d'amende pour s'être rendu coupable de 69 infractions au règlement torontois sur la pollution de l'eau, s'est vu infliger la sentence suivante pour outrage au tribunal: «One month in jail for every single day that he delays his decision to undertake a major works program to comply with the bylaw and the order of this Court - up to a maximum of a further fifteen months ${ }^{91}$.»

Par ailleurs, en 1986 toujours, l'ex-président de la compagnie Best Plating Shippe Limited (Scarborough, Ontario) M. Sam Siapa, a été condamné à six mois d'emprisonnement pour outrage au tribunal après avoir été reconnu coupable sous 49 chefs d'accusation et été condamné à $67950 \$$ d'amende92.

89. Commission de réforme du droit du Canada, Droit, objectifs publics et observation des normes, précité, p. 43.

90. Starr, Judson W., Countering Environmental Crimes, 1986, 13 B.C. Env't AFF Law Rev, p. 382.

91. La Reine c. Jetco Manufacturing Limited \& Keith Alexander, Toronto Weekly Court, Supreme Court of Ontario, RE 671-86, 21 mai 1986.

92. Lawyers Weekly, 22 août 1986. 
En droit fédéral, la compagnie Panartic Oils s'est vu imposer une sentence de $150000 \$$ d'amende et deux ans de probation, pour des infractions à la Loi sur la prévention de la pollution des eaux arctiques. Et le juge de conclure que les dispositions pénales doivent s'interpréter strictement: «In my judgment, dit-il, this court must not shy from its duty to tread where Parliament has provided, even if it involves imposing fines in amounts that are far beyond the range normally considerered by way of penalty in this court ${ }^{93}$.»

Quand le droit de l'environnement suggère des amendes minimales élevées et/ou des peines d'emprisonnement, il arrive de plus en plus souvent que les juges imposent des sanctions sévères aux corporations. En ce domaine, comme dans les autres domaines du droit, les juges appliquent d'abord la loi. Si cette loi prévoit une amende élevée, celle-ci sera vraisemblablement imposée ${ }^{94}$.

Certes, il ne faut pas minimiser les difficultés du procès, en particulier en ce qui concerne les exigences de preuve et les possibilités d'exonération en raison d'une défense de diligence raisonnable. Réunir des preuves de pollution exige un travail d'enquête suivi, que l'administration publique s'est révélée incapable d'assumer jusqu'à aujourd'hui, faute de volonté politique et faute d'un service des enquêtes.

La défense de diligence raisonnable permet au pollueur d'échapper à toute responsabilité pénale lorsqu'il démontre, à la satisfaction du juge, qu'il a pris toutes les précautions nécessaires pour éviter l'acte dommageable. Le pollueur bénéficie même d'un renversement du fardeau de la preuve, lorsqu'il démontre qu'il a pris quelques précautions raisonnables. C'est alors à la Couronne de prouver que ces précautions n'étaient pas suffisantes pour être qualifiées de «diligence raisonnable». Apparue dans notre droit depuis 1978, suite à un jugement de la Cour suprême en matière de pollution des eaux ${ }^{95}$, cette défense de diligence raisonnable s'est montrée au cours des années non seulement susceptible «d'interprétations contradictoires, à la pièce et incertaines", mais aussi source d'insécurité juridique pour l'administration, et partant, source d'inefficacité administrative ${ }^{96}$.

93. La Reine c. Panartic Oils Ltd., In re. the territorial of Court of the Northwest territories, Yellowknife, 14 janvier 1983.

94. Voir Swaigen, John et Gail Bunt, la Détermination de la peine en droit de l'environnement, précité.

95. Re La corporation municipale de Sault-Sainte-Marie, 1978 S.C.R. 1299.

96. V. Jacoby, Daniel et Gilles Létourneau, «Les soubresauts de Sault-Sainte-Marie et le droit pénal du Québec», $194141 R$ du B 459. 
On peut donc dire que ce ne sont pas les tribunaux qui freinent le développement du droit pénal de l'environnement. Au contraire, tout porte à croire que, devant des preuves manifestes et de nombreuses poursuites, les tribunaux n'hésiteraient pas à sanctionner sévèrement les infractions à l'environnement.

Ce qui freine le droit pénal de l'environnement, c'est d'abord le manque de volonté ferme des législateurs et des administrations publiques de sévir contre tous les pollueurs. Tant que cette volonté politique sera absente, certaines grandes entreprises continueront à détériorer impunément l'environnement.

Cette volonté ferme des législateurs et des administrations publiques doit se concrétiser dans le droit pénal de l'environnement, et être complétée par d'autres mesures, administratives, sociales ou civiles, afin de devenir véritablement dissuasive.

Le droit pénal de l'environnement ne doit donc pas être remis en cause. Plusieurs études récentes tendent à démontrer la nécessité des interventions pénales, pour réduire le nombre des infractions contre l'environnement: «Criminal actions authorized by environmental statutes have a much more deterrent effect than civil actions alone ${ }^{97}$.» Les grandes entreprises craignent en effet les retombées de la publicité négative due aux procès; elles s'inquiètent d'avoir à payer des amendes très élevées et d'encourir les foudres de l'opinion publique. Les entreprises qui contractent avec les gouvernements s'inquiètent des effets que peut avoir un dossier criminel sur le renouvellement de ces contrats. De plus, les poursuites pénales peuvent impliquer personnellement les administrateurs des compagnies; le paiement des amendes n'est pas assumé par les assurances et une éventuelle condamnation à l'emprisonnement en fait réfléchir plus d'un.

Un problème technique demeure, c'est celui de la détermination de la peine des grandes entreprises. À première vue, l'imposition d'amendes élevées peut sembler très dissuasive, mais ce sont souvent les détenteurs d'actions ou les consommateurs qui en assument les frais. De sorte que, pour atteindre minimalement ses objectifs de dissuasion, il faut toujours que cette amende soit plus élevée que les coûts estimés de la mise en place de dispositifs antipollution.

97. McMurry, Robert et Stephen D. Ramsey, «Environmental Crime: the Use of Criminal Sanctions in Enforcing Environmental Laws», Land Use and Environmental Law Review, 1987, p. 43659. 
Pour que ces entreprises se conforment aux lois et règlements, il faut aussi que l'amende excède les profits qu'elles comptent tirer, à long terme, de leurs activités illicites. Or, cela est impossible dans l'état actuel du droit de l'environnement, parce que le droit pénal statutaire n'a pas pour habitude de déterminer les sentences en fonction des contrevenants, mais en fonction des infractions.

D'autres moyens techniques doivent être examinés et ajoutés aux amendes pour obtenir la conformité des grandes entreprises aux lois et règlements. Parmi ceux-ci figurent la confiscation des biens, la suspension et la révocation des permis, l'emprisonnement, les ordonnances de probation, les injonctions, les mesures de restitution, de réparation et de dédommagement des victimes. Il faut aussi rechercher des solutions plus innovatrices, en dehors des mécanismes pénaux, tels la perception de droits d'effluents et d'une taxe sur la pollution, l'imposition d'amendes par les organismes administratifs ${ }^{98}$. De même qu'inventorier les possibilités qu'offrent, en matière pénale, les poursuites privées et l'action civile pénale 99 .

\section{CONCLUSION}

L'espace ne nous permet pas ici de tracer un bilan de l'utilisation du droit pénal de l'environnement, depuis 15 ans, pour proposer quelques avenues de réflexion et d'analyse critique. Rappelons toutefois, en guise de conclusion, que les Canadiens et Canadiennes acceptent de plus en plus difficilement les compromis en matière de santé publique et de protection de l'environnement. Le droit à la qualité de l'environnement fait maintenant partie des valeurs sociales fondamentales et, à ce titre, l'environnement réclame une protection accrue. Il faudra donc, tôt ou tard, que les gouvernements révisent leurs politiques de soutien quasi inconditionnel à l'industrie en tenant compte

98. Swaigen, John et Gail Bunt, la Détermination de la peine en droit de l' environ nement, précité. p. 84 ss.

99. Tel que de cela existe en France, par exemple. Jacques-Henri Robert et Martine Rémond-Gouilloud, Droit pénal de l'environnement précité, p. 272. 